\title{
THE SHARED-LOAD WEAR MODEL IN LUBRICATED SLIDING: SCUFFING CRITERIA AND WEAR COEFFICIENTS*
}

\author{
YOUNG-ZE LEE and K. C. LUDEMA \\ Mechanical Engineering Department, University of Michigan, Ann Arbor, MI 48109-2125 \\ (U.S.A.)
}

(Received May 16, 1989; accepted September 4, 1989)

\section{Summary}

One model that is offered for predicting the wear of minimally lubricated sliding parts suggests that wear may be predicted directly from the mechanics of contact. In this model the lubricant is assumed to carry part of the applied load and asperities carry the remainder of the load by solid contact and adhesion with each other.

Experiments show that the assumed metallic adhesion does not take place on the predicted scale in ordinary practice, and thus catastrophic wear does not necessarily take place. The reason is that protective chemical species form on the asperities to prevent high surface shear stress. These films form in all practical systems but are particularly effective when a proper running-in procedure is followed.

\section{Introduction}

Lubricated sliding components such as gears and cams sometimes fail catastrophically and without warning. This sudden mode of failure is often called scuffing, or scoring, or some combinations of these terms.

Proper break-in can prevent scuffing in fairly severe service but little is known of the best way to break in surfaces. The incentive in studying breakin is that "broken-in" surfaces can sustain up to ten times the load that a new surface can and function for an adequate period of time.

Most of the early studies in scuffing focused on the conditions of the lubricant for preventing scuffing, ignoring changes in surface roughness during a test. Several papers from such studies suggest that, when the surfaces of sliding members or the lubricant between them reached a particular temperature, then scuffing would occur $[1,2]$. Later papers discussed the role of lubricant film thickness in scuff initiation [3,4]. Attention was drawn to the comparison of lubricant film thickness with the height of

*Paper presented at the International Conference on Wear of Materials, Denver, CO, U.S.A., April $8 \cdot 14,1989$. 
asperities. A very useful ratio $\Lambda$ consists of $h / \sigma$, where $h$ is the film thickness and $\sigma$ is the composite surface roughness of the two contacting surfaces. Generally, it could be supposed that a value of $\Lambda$ greater than 1 would imply that (statistically) few asperities would contact each other, which would suggest that little or no wear should take place. In conventional thinking, values of $\Lambda$ less than 1 should be avoided because asperity "contact" would lead to high wear, and possibly scuffing. This may be implied from most mechanics-based models for wear, since the staple in most such works is the expression that, when asperities contact each other, "there is adhesion" and with adhesion there should be junction growth.

In practical machinery, however, there are many examples of surfaces that survive values of $\Lambda$ as low as 0.3 . No theoretical explanation has been offered for these observations but surface survival is connected with the formation of non-metallic species that form on the sliding surfaces from oxygen and other compounds in the lubricant. More recently it has been found that the materials and surface roughness of the sliding members were as important as the lubricant $[4,5]$. $\mathrm{Fe}_{3} \mathrm{O}_{4}$ was found to form on surfaces lubricated with mineral oil. The exact composition of films formed in engine oil has not been determined. It has also been shown that material properties, material microstructure, duty cycle of sliding and several other variables are as important as surface temperature $[6,7]$. Further, it appears that there is an optimum value of $\sigma$ at which scuffing resistance exceeds that for very small values of $\sigma$.

Few papers discuss the wear rate of surfaces in lubricated sliding, much less the mechanisms of wear in that regime. One of the more carefully developed models for the wear of lubricated sliding systems is the shared load wear model of Tallian et al. [8]. In this model, part of the applied load across a sliding boundary is carried by the lubricant trapped between the surfaces, and part of the load is carried by asperities that stand higher than the thickness of the lubricant film. Wearing takes place in proportion to the fraction of load carried by the asperities. The lubricant film thickness is calculated with the equations of elastohydrodynamics and the asperities are described in the terms of the Williamson-Greenwood statistical formulations $[9-11]$.

In the literature on scuffing, there has been little or no consideration of time-dependent phenomena in scuffing and break-in. Early during sliding in some environments a soft film forms, over time, on the sliding surface and often the surface roughness changes. Both of these changes affect friction, and a change in surface roughness influences $\Lambda$. This paper reports the results of an exploration of several time-dependent events in lubricated sliding where $\Lambda$ ranges from about 3 to 0.005 . These include surface roughness and contact resistance, from which real contact area may be inferred. The experiments described below also emphasize the wear rates of sliding surfaces, comparing them with those published under the heading of the shared load wear model, and connecting them with the physical and chemical events on sliding surface. 


\section{Experiments}

The experimental apparatus was the cylinder on plate configuration, previously reported, and shown in Fig. 1. The stationary (non-rotating) cylinder is a crowned roller made of 52100 steel with a Rockwell $\mathrm{C}$ hardness of $57 \mathrm{HRC}$. The cylinders are $6.35 \mathrm{~mm}$ diameter and $6.35 \mathrm{~mm}$ long. The plates were 1020 steel, were oil quenched from $750{ }^{\circ} \mathrm{C}$ and have a hardness of $8 \mathrm{HRC}$. These were polished in random directions, producing a surface roughness $R_{\mathrm{q}}$ of $0.032 \mu \mathrm{m}$. Three lubricants were used, namely a laboratory grade of mineral oil of 125 - 135 cSt viscosity, a commercial "synthetic" automotive engine oil with additives, and a vacuum pump oil, all of nearly the same viscosity at $20{ }^{\circ} \mathrm{C}$. The latter was used in a nitrogen atmosphere, and the others were used in air.

The electrical contact resistance was measured in order to come to some conclusion on the real area of contact, as was done by Tallian and coworkers $[8,12]$. However, usually electrical contact resistance is measured as if there are two states, metallic contact and "no" contact [12]. Thin films of lubricant have an electrical resistance of the order of a few hundred milliohms whereas metallic contact would have resistivities several orders lower. The contact resistance between these values would imply partial asperity contact, but it is a very difficult quantity to measure. In the present experiments, intermediate values of contact resistance were measured with the circuit shown in Fig. 2.

Most tests were done by beginning at the high $\Lambda$ value of about 3.0 and progressing stepwise to lower values of $\Lambda$. Recall that $\Lambda=h / \sigma$ where $h$ is the fluid film thickness and $\sigma=\left(\sigma_{1}{ }^{2}+\sigma_{2}{ }^{2}\right)^{1 / 2}$ in which $\sigma_{1}$ is the surface roughness $R_{\mathrm{q}}$ of one surface, etc. In order to progress toward lower values of $\Lambda$, it was therefore necessary to measure $R_{\mathrm{q}}$ before each test and to adjust the loads and speeds to achieve the desired value of $\Lambda$. The loads and speeds were determined from the equation of elastohydrodynamics for a cylinder sliding on a flat plate to obtain the fluid film thickness $h[10]$ :
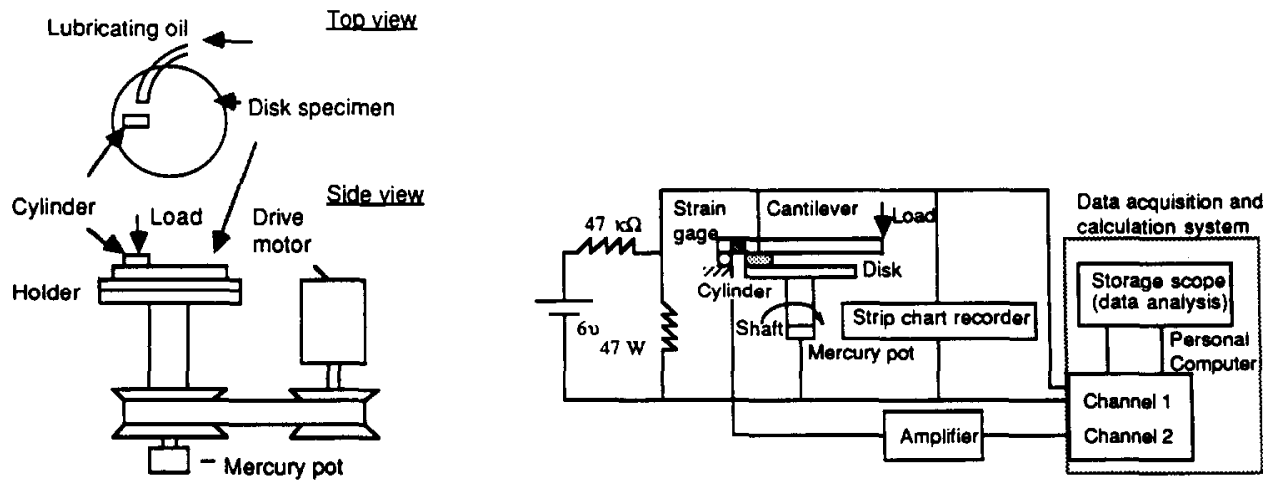

Fig. 1. Schematic diagram of the wear tester.

Fig. 2. A system for measurement of electrical contact resistance. 
$\frac{h}{R_{\mathrm{x}}}=2.69 U^{0.67} G^{0.53} W^{-0.067}\left(1-0.61 \mathrm{e}^{-0.73 k}\right)$

where $U=\mu V / E^{\prime} R_{\mathrm{x}}, G=\alpha E^{\prime}, E^{\prime}=E /\left(1-\nu^{2}\right)$ and $\mu=\gamma \rho . W$ is the applied load, $R_{\mathrm{x}}$ is the radius of the roller, $V$ is the sliding speed, $\gamma$ is the kinematic viscosity of the lubricant, $E$ is Young's modulus, $\nu$ is Poisson's ratio, $\rho$ is the density of the lubricant and $\alpha$ is the pressure-viscosity index of the lubricant.

Each test was run for $3 \mathrm{~min}$ at one value of $\Lambda$, after which the surface roughness was measured with a surface tracer system. A new load and speed were selected to obtain the next lower value of $\Lambda$ for the next $3 \mathrm{~min}$ of testing. These were simply referred to as the progressive loading tests although other changes took place as well.

The amount of wear from the sliding track on the plate was very small and was determined by an expanded trace across the track on a strip chart from the surface tracer system.

Some tests were done under conditions for $\Lambda \ll 1$ without break-in in order to determine whether these surfaces would survive more than $15 \mathrm{~min}$. These are referred to as the immediate loading tests. The selection of $15 \mathrm{~min}$ survival is arbitrary.

\section{Results}

\subsection{Changes in surface roughness}

In the progressive loading tests the surfaces all became rougher. This is not the case with all tests of this kind but is rather the case with steel of $8 \mathrm{HRC}$ hardness with a surface roughness of $0.032 \mu \mathrm{m}$. The values of surface roughness in $R_{\mathrm{q}}$ (r.m.s. roughness) are given in Fig. 3(a). These values were included in the calculation of $\Lambda$ for the next experiment as shown in Fig. 3 (b). It should be noted that, in the progression of the tests, the same values of $\Lambda$ were not always used with each lubricant. Rather, from preliminary
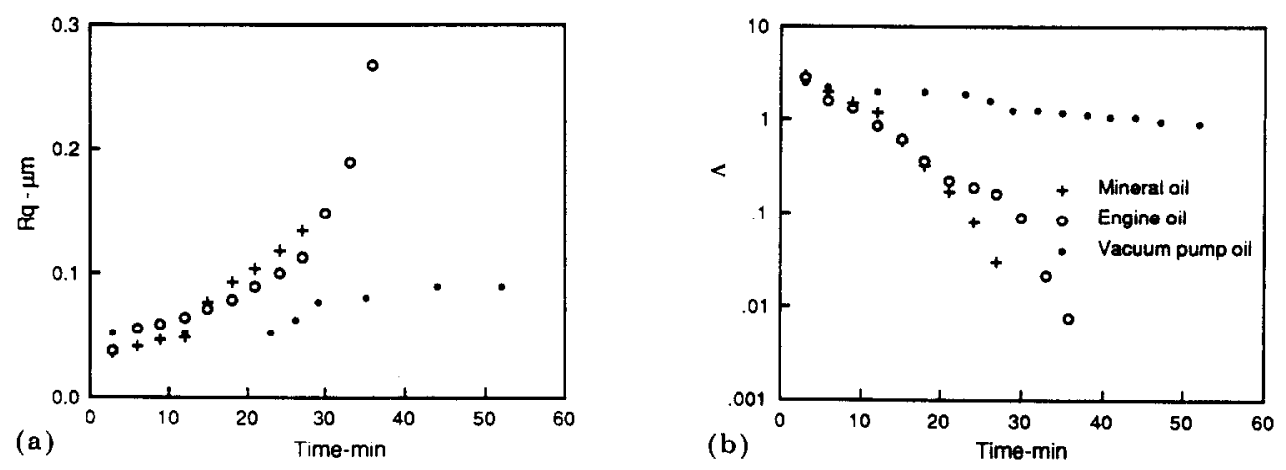

Fig. 3. The changes in (a) surface roughness $R_{\mathrm{q}}$ and (b) $\Lambda$ during the tests. 
experiments it was found that the vacuum pump oil would not adequately lubricate at $\Lambda$ less than 1 whereas the other lubricants did. Thus the range of $\Lambda$ over which the three lubricants would function was divided into about 12 or 15 steps as shown in Fig. 3 for each of the lubricants.

\subsection{Contact resistance}

Changes in the contact resistance $\Omega$ in the tests are shown in Fig. 4 . These tests, again, began with the highest values of $\Lambda$. The data for the vacuum pump oil under nitrogen cover gas are a straight line to the failure point at about $\Lambda=0.9$. The data for the other lubricants are parallel to that of the vacuum pump oil but levels off at about $1 \Omega$. Surely the real loadcarrying area, i.e. the summation of the small asperity contact "points", increases as $\Lambda$ decreases and $\Omega$ should accordingly decrease. The plateau probably indicates that any tendency for $\Omega$ to decrease because of an increasing contact area is offset by the increased resistance due to thicker surface films. Very probably these same films offer protection from scuffing.

\subsection{Wear rate for sliding surfaces}

The wear rate of the 1020 steel plate when using the three lubricants is shown in Fig. 5. The tests using the vacuum pump oil under nitrogen cover gas ended at $\Lambda \approx 0.9$ as noted before, but the wear rate is linearly related to $\Lambda$ as Tallian et al. [8] also found. Their data are reproduced in Fig. 5 for comparison. The curves for the wear rates of the other two lubricants include linear parts that extend well into the region below $\Lambda=1$ and change slope at still more severe conditions. It should be noted that the initial slopes
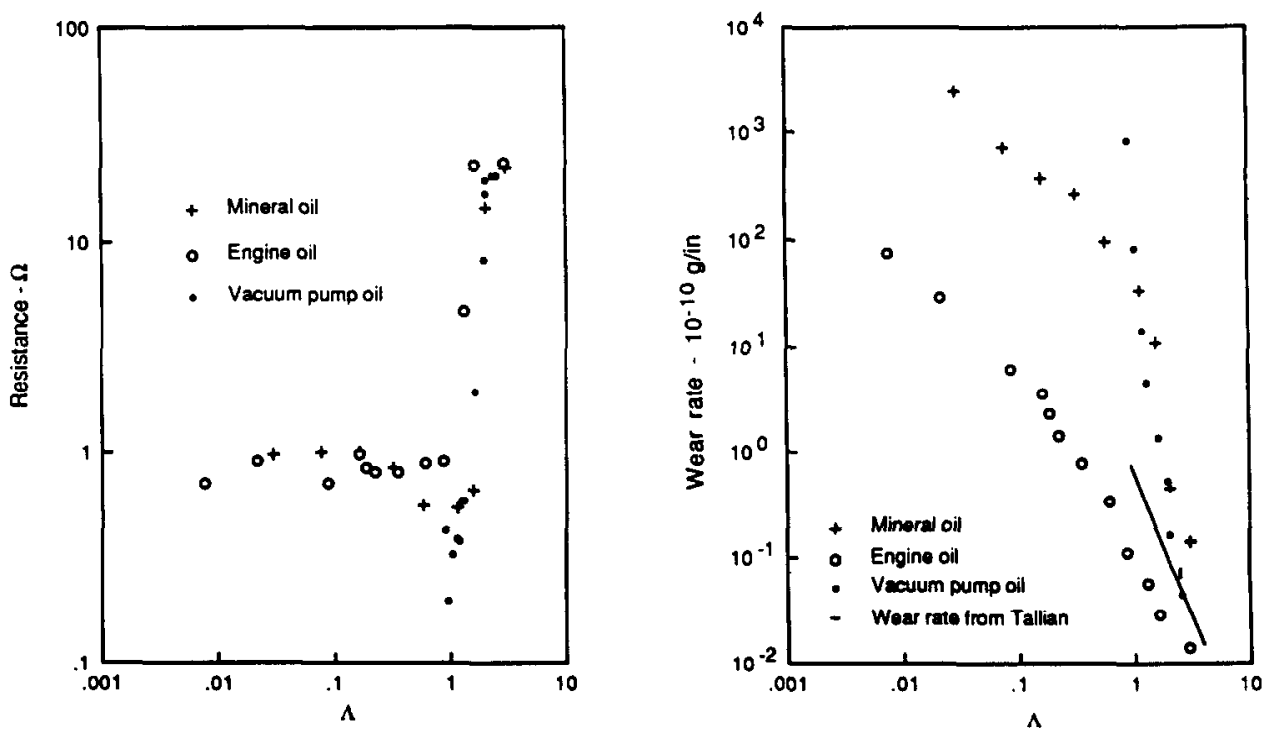

Fig. 4. Variation of the contact resistance with $\Lambda$.

Fig. 5. Wear rate us. dimensionless film thickness $\Lambda$. 
of these three curves are each different. This would strongly suggest that the exponents on $W$ and $V$ in the Archard equation for wear rate should be some value other than $1[12]$.

\section{Discussion}

Tallian et al. [8] assumed that the wear rate is proportional to the real contact area and they calculated the real contact area using the WilliamsonGreenwood equations [9-11], which are based on the assumption that asperities of random height and shape deform plastically as two surfaces are pressed together. Tallian et al. adjusted the load on the asperities and the amount of interference between asperities by calculating the thickness and load-carrying ability of the liquid film between the two surfaces. They finally calculate the ratio $A_{\mathrm{c}} / A_{0}$, where $A_{\mathrm{c}}$ is the asperity contact area and $A_{0}$ is the lubricant wetted area, for various values of $\Lambda$. Their results are shown in Fig. 6. Values of $A_{\mathbf{c}} / A_{0}$ vs. $\Lambda$ were also calculated from the surface roughness values of the specimens in the present research and these are also plotted in Fig. 6. These values are near those calculated by Tallian $e t$ al.

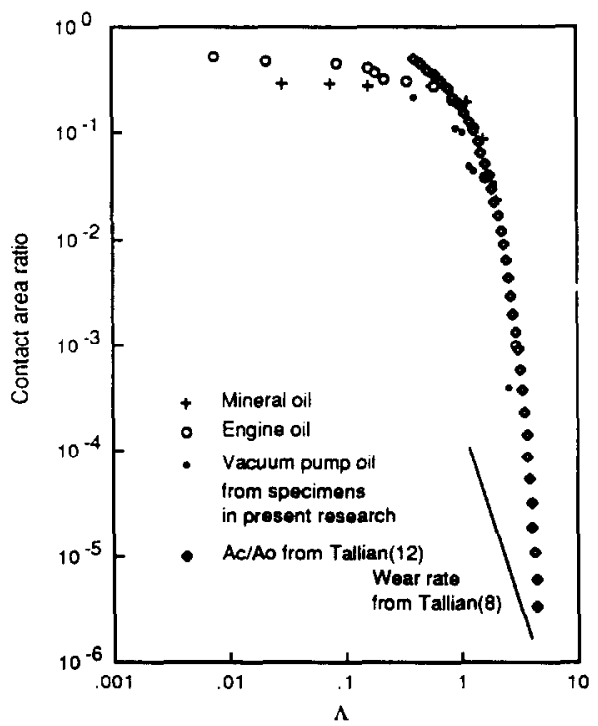

Fig. 6. Contact area ratio us. dimensionless film thickness $\Lambda$.

Figure 7 is a plot of the wear rate data and the data of $A_{0} / A_{0} v s . \Lambda$ of Tallian et al. Whereas the shapes of some curves are similar, the numerical values and slopes are not sufficiently similar to say that the theory of Tallian et $a l$. is adequate for predicting wear rate in lubricated sliding. Further, we have taken the curves for contact resistance from Fig. 4, inverted and adapted them to the axes of Fig. 7 in Fig. 8. This was done because it is 


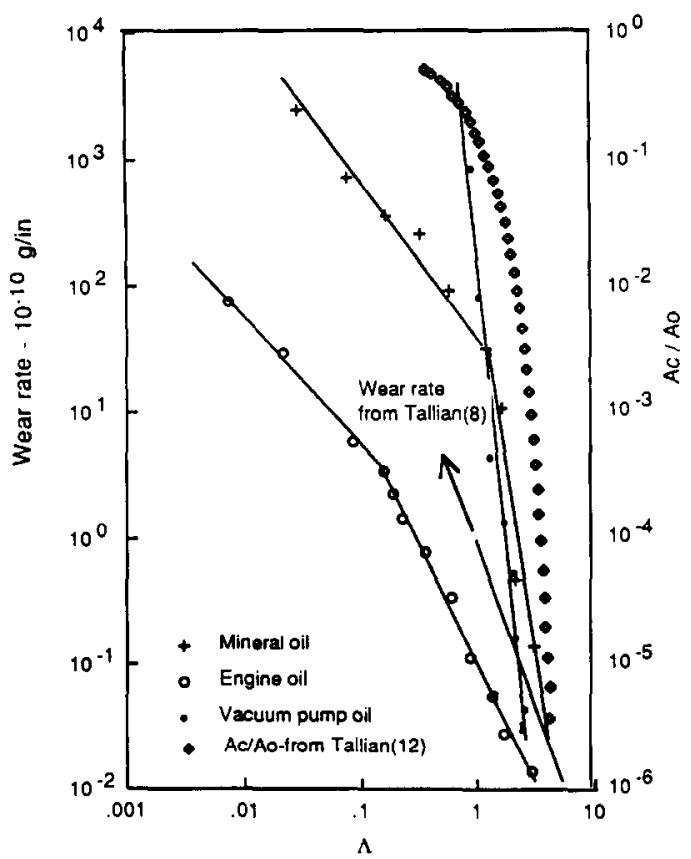

Fig. 7. Wear rate and ratio of contact area from the work of Tallian et al. vs. dimensionless film thickness $\Lambda$.

frequently assumed that the reciprocal of contact resistance should be a measure of real area of contact, which, again, should correlate with wear rate. The disparity in the results is about four orders of magnitude (not different scales) and in addition the contact resistance curve has a horizontal section where the curves of wear rate have negative slopes. From this plot it can be seen that the contact resistance may indicate differing natures of contact, metallic vs. non-metallic coating but is not useful for indicating wear rate. It appears that a non-conducting and perhaps protective chemical film was formed more readily from the additives in the engine oil than from the constituents in the other oils, and the vacuum pump oil contained few or no species for producing a protective film. It appears also as if the mineral oil used by Tallian et al. may have contained considerable amounts of surface active constituents. They were clearly not working under conditions described by classical laws of "adhesive wear".

Finally, an attempt was made to determine how long a lubricated surface would survive when loads were applied by two methods, namely by immediate loading and by progressive loading, both at constant speed. Figure 9 is the plot of survival time using mineral oil as the lubricant. The vertical axis is the applied load of hertzian contact stress. At $200 \mathrm{~N}$ the survival time with progressive loading is 20 times that with immediate loading. At $800 \mathrm{~N}$ there is a ten-fold difference, and at $1500 \mathrm{~N}$ the difference is only four times. The survival times for both tests converge to one point at a sufficiently high load. At this point the average contact stress is calculated to be 


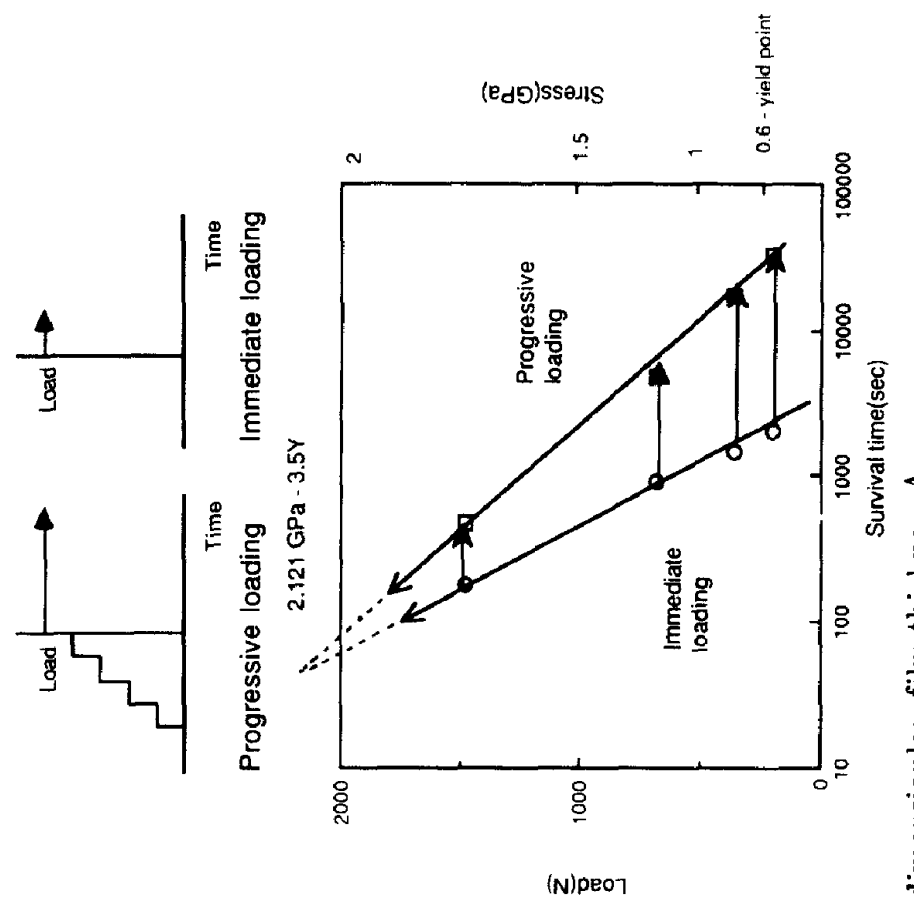

U/I

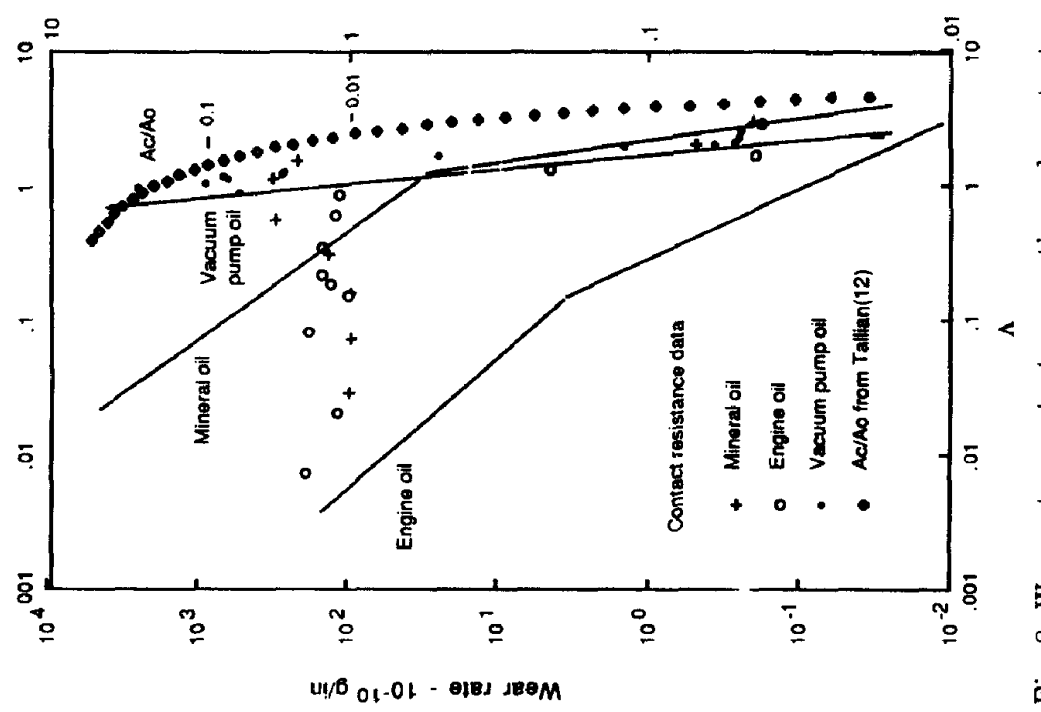

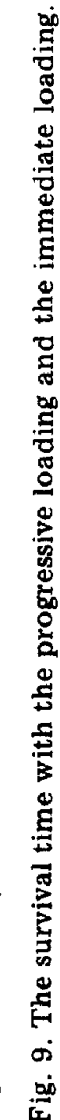


3.5 times the yield strength of the specimen. It is seen that, with progressive loading, there was a time for a protective layer to form on the sliding surface but not with immediate loading. We conclude that the difference between the survival times of the two loading methods indicates the efficiency of break-in. With mineral oil the benefit of a break-in procedure by progressive loading diminishes as load increases, until no break-in procedure is effective at all.

\section{Conclusions}

(1) Scuffing does not necessarily occur when the lubricant film thickness is much smaller than the composite surface roughness. Surfaces survive at values as low as $\Lambda=0.005$, provided that some sliding first occurs at larger values of $\Lambda$ and there are chemically active species in the lubricant.

(2) Predictions of wear rate based on the adhesion theories of contact and friction may apply to cases of unoxidized and uncoated steels but not to more practical systems.

(3) Wear coefficients based on the Archard wear equations for lubricated sliding are not simply some multiple of that for dry sliding [13]. This is indicated by curves instead of straight lines in Fig. 5 .

(4) Predictions of the tendency for scuffing cannot be based on initial values of $\Lambda$ because surface roughness changes during sliding.

(5) The benefit of a break-in procedure diminishes at higher load and there appears to be a point at which no break-in procedure is effective. The calculated average contact stress at this point is 3.5 times of the yield strength of the specimen.

\section{References}

$1 \mathrm{H}$. Blok, Theoretical study of temperature rise at surface of actual contact under boundary lubrication contacts, Inst. Mech. Eng. Proc., 3 (1937) 438.

$2 \mathrm{P}$. M. Ku, Gear failure modes - importance of lubrication and mechanism, ASLE Trans., 19 (1976) 239.

3 A. Dyson, Scuffing review, Tribol. Int., 8 (1975) 77, 117.

4 Q. J. Xue and $K$. C. Ludema, Plastic failure effect in scuffing of soft metals, in K. C. Ludema (ed.), Proc. Int. Conf. on Wear of Materials, American Society of Mechanical Engineers, New York, 1983, p. 499.

5 G. R. Miller, J. M. Powers and K. C. Ludema, Frictional behavior and surface failure of dental feldspathic porcelain, Wear, 31 (1975) 307 - 316 .

$6 \mathrm{~K}$. C. Ludema, A review of scuffing and running-in of lubricated surfaces, with asperities and oxides in perspective, Wear, 100 (1984) 315.

$7 \mathrm{M}$. Suzuki and K. C. Ludema, The wear process during the running-in of steel in lubricated sliding, ASME Paper 86-Trib-44 (American Society of Mechanical Engineers, New York).

8 T. E. Tallian, J. I. MeCool and L. B. Sibley, Partial elastohydrodynamic lubrication in rolling contact, Inst. Mech. Eng. Proc., 180 (3B) (1965 - 1966) 169 - 184. 
9 K. L. Johnson, J. A. Greenwood and S. Y. Poon, Asperity contact in elastohydrodynamic lubrication, Wear, 19 (1972) 91.

10 B. J. Hamrock and D. Dowson, Isothermal elastohydrodynamic lubrication of point contacts, Parts I and II, J. Lub. Tech., 98 (1976) 223, 275.

11 J. I. McCool, The distribution of microcontact area, load, pressure, and flash temperature under the Greenwood-Williamson model, J. Lub. Tech., 110 (1988) 106.

12 T. E. Tallian, The theory of partial elastohydrodynamic contacts, Wear, 21 (1972) 49.

13 M. B. Peterson and W. O. Winer (eds.), Handbook of Wear Control, American Society for Mechanical Engineers, New York, 1980. 